\title{
Stress induced changes in hydrogen pressure in contact with tubular diffusion membrane
}

\author{
K Kandasamy* \\ Department of Physics, University of Jaffna
}

\begin{abstract}
Hydrogen permeation studies in Palladium and Palladium alloys reveal that hydrogen diffusion is non-Fickian in metals and their alloys due to the stress field induced by the hydrogen in their lattice. Previously the non-Fickian, uphill diffusion of hydrogen in $\mathrm{Pd}_{81} \mathrm{Pt}_{19}$ alloy membrane was explained by attributing it to the modification in the space-time variation of the concentration of dissolved hydrogen in the metal lattice due to Diffusion-Elastic effect. This paper reports evidence for the combined effect on the space-time variation of the concentration of dissolved hydrogen in the lattice by the Gorsky effect and the Diffusion -Elastic effect when hydrogen diffusion initiated in the diffusion membrane either by suddenly increasing or reducing the hydrogen pressure at one wall of the membrane.
\end{abstract}

Key words : Diffusion-Elastic effect, Gorsky effect, hydrogen absorption, Joule-Thomson effect, metal- hydrogen system, Non-Fickian Diffusion

\section{Introduction}

The diffusion of hydrogen in metals has been a long standing fundamental research study due to its uniqueness. The effects of hydrogen on the properties of metals and their alloys used in industries are of great importance due to steady absorption of hydrogen by metals and their alloys in ambient conditions and the presence of hydrogen in the atmosphere. Further chemical simplicity of hydrogen allows understanding the underlying physical natures of metals and their alloys that drive the diffusion of hydrogen in their lattices. In other words, the hydrogen diffusion studies have both academic and technical interest. In the past, new sets of research data observed for hydrogen diffusion in tubular membranes of palladium and its alloys were published by Lewis and their collaborates (Lewis et al., 1983, 1987, 1988, Baranowski et al., 1989 and Sakamoto et al.,1996). The typical features of the observed time dependent changes of hydrogen pressure inside the tubular membrane due to the commencement of hydrogen diffusion either by gas adsorption or electrolysis are shown in Figures $1 \mathrm{a}$ and $1 \mathrm{~b}$.

* Correspondence: profkandasamy@gmail.com 


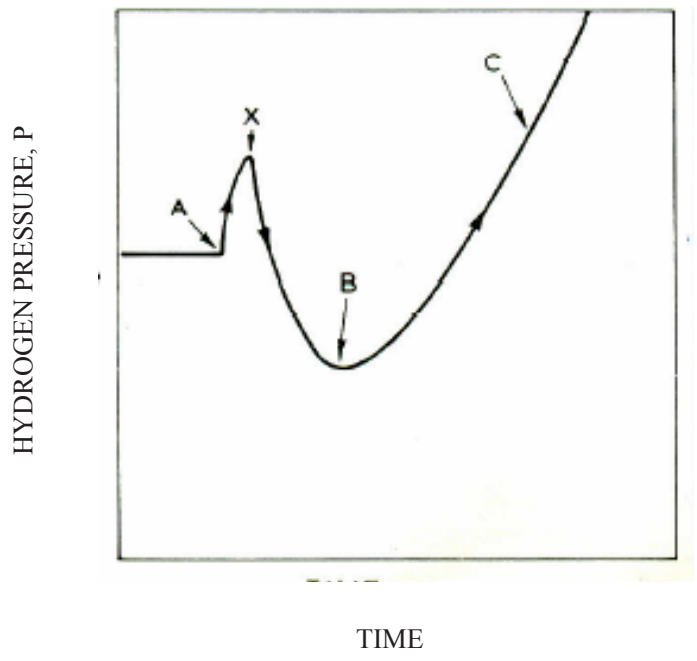

Figure 1a: Time dependent changes, from an initially virtually constant pressure (Po) of hydrogen gas within a tubular membrane, following a sudden increase of the hydrogen pressure at the outer wall of the tubular membrane (i.e. commencement of hydrogen diffusion by gas adsorption).

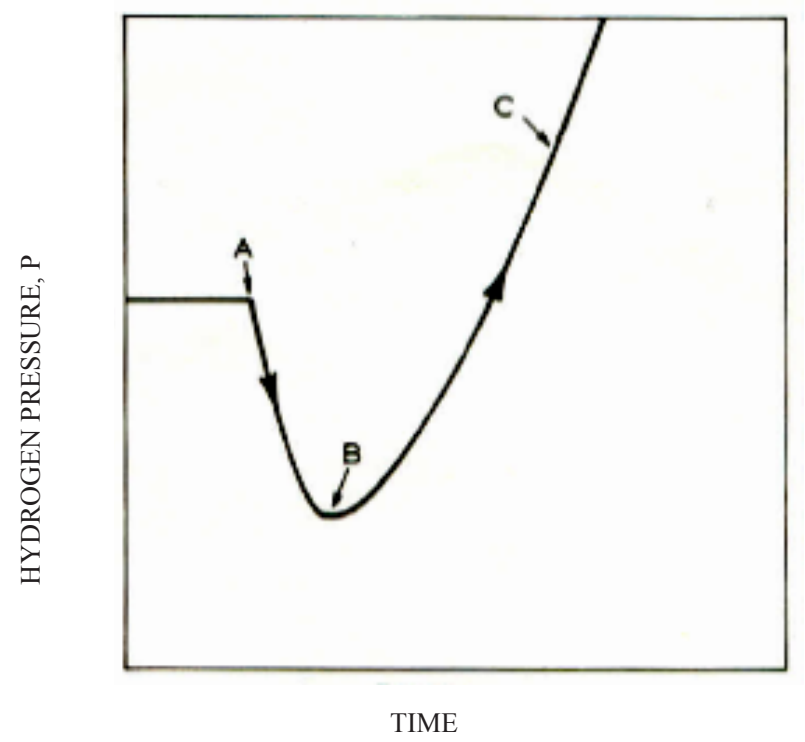

Figure 1b: Time dependent changes, from an initial virtually constant pressure (Po) of hydrogen gas within a tubular membrane, following an increase of the hydrogen content of the outer wall of the tubular membrane (i.e commencement of hydrogen diffusion by electrolytic cathodisation). 
The observed diffusion of hydrogen against concentration gradient of hydrogen in the diffusion membrane (uphill diffusion) was explained by Kandasamy (Kandassmy 1988). However the initial time dependent changes of hydrogen pressure inside the tubular membrane (part AX in figure 1.a) and the difference between the observed results at the initial stage of the experimentation when hydrogen diffusion commenced in tubular membranes by electrolysis and gas phase adsorption are not accounted. The difficulty in accounting for the initial time dependent changes hydrogen pressure arises due to the coupling of diffusion and elastic fields in the diffusion membrane. An elastic field in a diffusion membrane can originate either by mechanically induced lattice strain (Gorsky effect) or by diffusion induced lattice strain (Diffusion -Elastic effect) or combination of both.

\section{Materials and Methods}

In the diffusion experiments tubular membranes made of Palladium, and Palladium alloys $\left(\mathrm{Pd}_{81} \mathrm{Pt}_{19}\right.$ and $\left.\mathrm{Pd}_{77} \mathrm{Ag}_{23}\right)$ were used. The hydrogen diffusion was commenced by introducing hydrogen to the outer surface of the tube membrane either by electrolysis (Lewis et al., 1987, Kandasamy et al., 1991) or gas phase adsorption (Kandasamy et al., 1990). The pressure changed inside the tubular membrane were recorded by a pressure gauge. The recorded pressure changes corresponding to various experimental conditions are presented in Figure 2 and Figure 3.

\section{Results and Discussion}

The initial pressure drop in the tubular membrane on cathodization and the initial pressure increase in the tubular membrane on anodization were previously explained by Kandasamy (1988) and Simon et al., (1991). But the initial increase of hydrogen pressure in the tubular membrane following a sudden increase of the hydrogen pressure at the outer surface of the wall of the tubular membrane and the initial drop in hydrogen pressure in the tubular membrane following a sudden reduction in hydrogen pressure at the outer surface of the wall of the tubular membrane are not observed either in the cathodization or in anodization experiment.

\section{Pressure changes due to volume cha nges}

The initial changes of pressure within the tubular membrane on sudden alteration of pressure at outer surface of the tubular membrane area are new and somewhat surprising observation. Initially it was thought that these changes in the hydrogen pressure within the tubular membrane was due to changes in the internal volume of the tubular membrane as a result of bending of tube wall on sudden increase or reduction in hydrogen pressure at the outer surface of the tubular membrane as shown in Figure 4. 

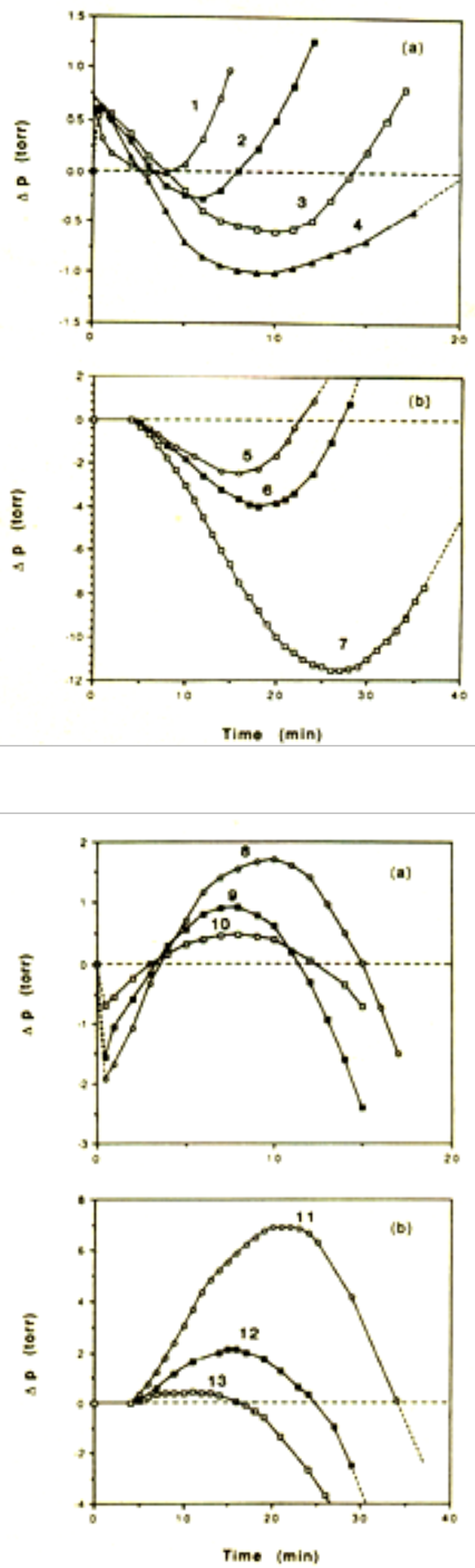

Figure 2:

(a) Time dependences of incremental changes of hydrogen gas pressure $\Delta p$ within a tubular $\mathbf{P d}_{81} \mathbf{P t}_{19}$ membrane, from initial pressures Po of 25 Torr (curve 1), 300 Torr (curve 2). 600 Torr (curve 3) and 740 Torr (curve 4) following increases of hydrogen pressures at the outer surface to 940 Torr (curves 1, 3 and 4) and 925 Torr (curve 2) $25^{\circ} \mathrm{C}$.

(b)Time dependences of incremental changes of $\Delta p$ from initial quasi-static pressures Po of 18,5 Torr (curve 5), 325 Torr (curve 6) and 650 Torr (curve 7) following recommencement of cathodization at outer surfaces at a current density of $15 \mathrm{~mA} \mathrm{~cm}^{-2}\left(25^{\circ} \mathrm{C}\right)$.

Figure 3:

(a) Time dependences of incremental changes of hydrogen pressure $\Delta \mathrm{p}$ within a $\mathbf{P d}_{81} \mathbf{P t}_{19}$ membrane from initial pressures Po of 45 Torr (curve 10), 640 Torr (curve 9) and 825 Torr (curve 8) following sustained evacuative removal of hydrogen from the outer surface $\left(25^{\circ} \mathrm{C}\right)$.

(b) Time dependences $\Delta p$ from initial quasi-static pressures poof 240 Torr (curve 13), 510 Torr (curve 12) and 720 Torr (curve 11) following commencements of anodizations of outer surfaces at a current density of $15 \mathrm{~mA} \mathrm{~cm}^{-2}\left(25^{0} \mathrm{C}\right)$. 

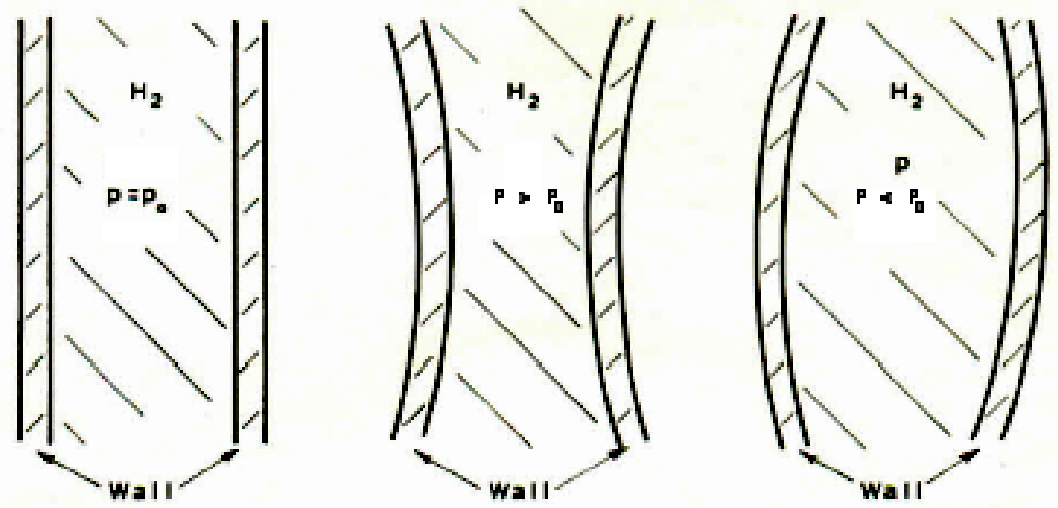

Figure 4: A diagrammatic comparison of the shape of the wall of a tubular membrane on sudden increase or reduction in hydrogen pressure, $P$ at the outer surface of the tubular membrane.

However a complementary experiment with argon gas to compare the internal volume changes on the sudden increase or reduction in argon gas pressure at the outer surface of the tubular membrane showed that these changes are too small compared to the changes observed in experiments with hydrogen gas. Further direct measurements of actual volume changes in complementary water displacement studies also show that the actual volume change is very small supporting the results of the experiments conducted with argon gas. Therefore the initial pressure changes observed with hydrogen cannot be accounted by volume changes of the tubular membrane. Further to study the possible influence of the Joule-Thomson effect in the pressure change on the sudden introduction of hydrogen or argon gas to the outer wall of the tubular membrane, the space surrounding the tubular membrane was filled with high heat capacity materials such as copper sheets but no significant influence was observed. Therefore the initial changes in the hydrogen pressure in the tubular membrane are due to hydrogen diffusion in the membrane.

\section{Competition between Gorsky effect and Diffusion-Elastic effect}

The modification in the concentration of dissolved hydrogen in metal lattice under the influence of tensile stress, $\sigma$ is expressed (Flanagan et al., 1976, Chen Min Li, 1978) as $\mathrm{C}(\sigma)=\mathrm{C}(0)$ $\exp (\mathrm{V} \sigma / \mathrm{RT})$, where $\mathrm{C}(0)$ is the concentration of dissolved hydrogen when the metal lattice is stress free and $\mathrm{V}$ is the partial molar volume of hydrogen in metals $\left(\sim 10^{-6} \mathrm{~m}^{3} / \mathrm{mole}\right)$. In these experiments since the tensile stress, $\sigma$ is equal to the applied pressure difference $\Delta \mathrm{P}$ and the term $(\mathrm{V} \sigma / \mathrm{RT})$ is very small, hence the changes in the concentration of dissolved hydrogen in metal lattice is proportional to PABased on the pressure-concentration isotherm for these alloys the change is expected to be proportional to applied pressure difference. This suggests that 
the experimentally observed pressure changes are not purely due to applied stress i.e. Gorsky effect.

The space-time variation of stress induced in the metal lattice by the hydrogen absorption in the metal lattice and followed by diffusion is shown in figure 5 (Kajenthini, 2012) and the space time variation of hydrogen concentration on the commencement of hydrogen diffusion by hydrogen absorption is shown in figure 6 (Kandasamy, 1995).

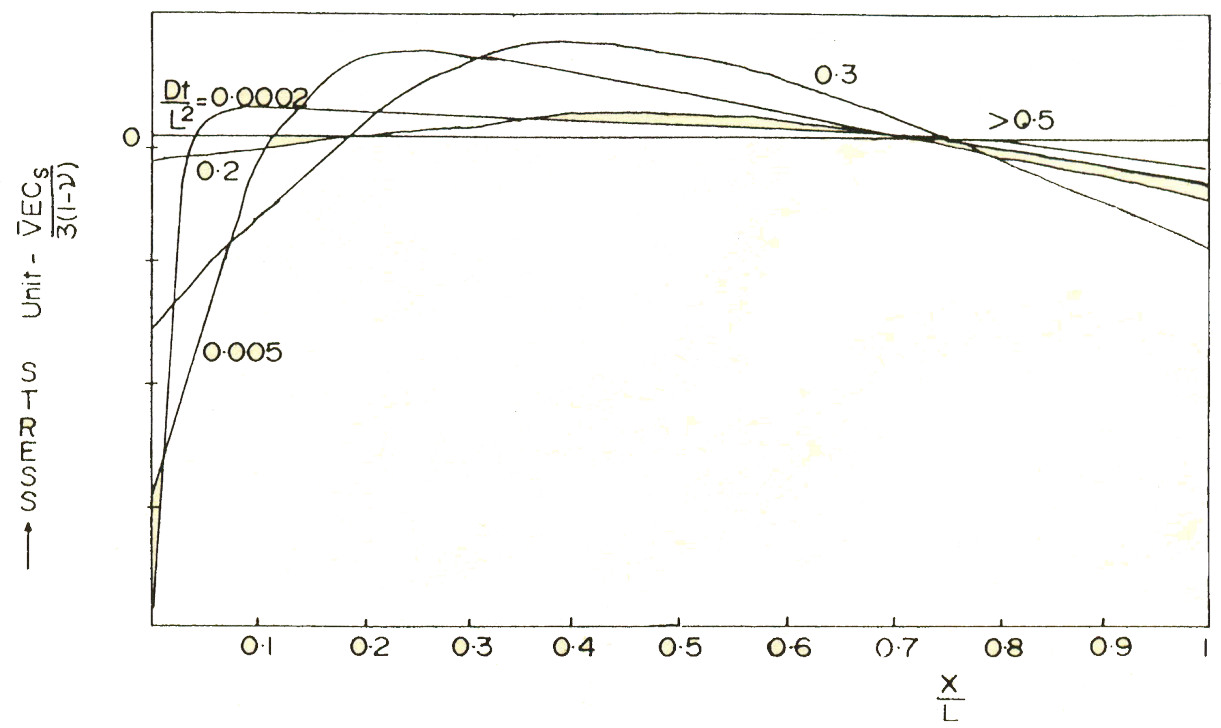

Figure 5: Space-time variation of stress components induced by the diffusion of hydrogen in the diffusion membrane.

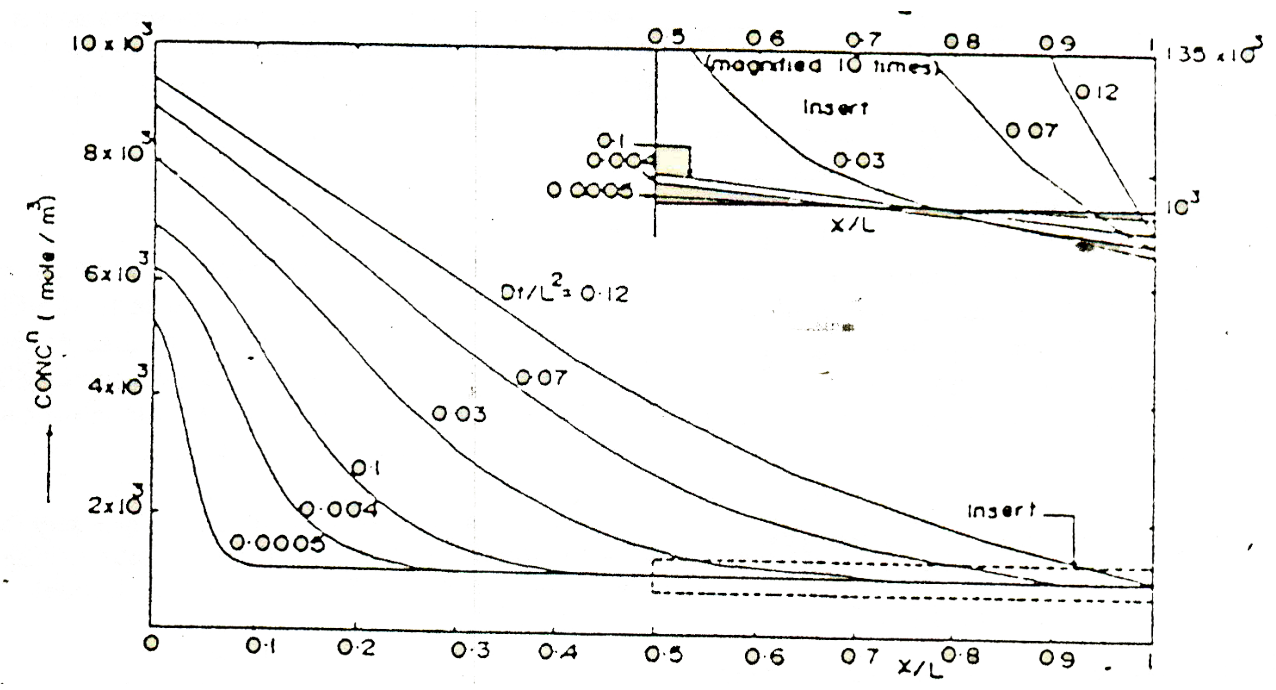

Figure 6. Space time variation of hydrogen concentration in the diffusion membrane 
The corresponding space-time variation of hydrogen concentration in diffusion membrane is shown in figure 6 . The space-time variation of the induced stress and hydrogen concentration suggests that the diffusion-elastic effect on the concentration of dissolved hydrogen in the metal lattice is also present simultaneously with the Gorsky effect. Therefore the initial changes of pressure in the tubular membrane is the combined result of the Gorsky effect and Diffusion-elastic effect. The former increases the pressure in the tubular membrane and latter reduces the pressure in the membrane. The pressure changes suggest that initially the Gorsky effects dominates and later diffusion-elastic effect gradually starts to dominate and changes the direction of pressure change as experimentally observed.

\section{Absence of initial pressure increase in cathodization or drop in anodization experiments}

The above explanation suggests absence or ineffectiveness of Gorsky effect contribution to changes in pressure in electrolysis experiments. In cathodization experiments hydrogen ions are produced in acid medium and drifting is towards the tubular membrane which act as cathode. In anodization the reverse take in place. It appears that these chemical processes are not capable of producing hydrostatic pressure at the wall of the tubular membrane to produce Gorsky effect . Hence in electrolysis experiments initial changes in pressure in the tubular membrane is purely due to diffusion-elastic effect.

\section{Conclusion}

The hydrogen diffusion process in thin metallic membranes seems to be governed by the combination of (a) imposed concentration gradient across the membrane, (b) diffusionElastic effect initiated by the inclusion of hydrogen during the diffusion process and (c) Gorsky effect due to the applied hydrogen pressure difference across the membrane. As a consequence of this the estimated diffusion coefficient of hydrogen, DH in metallic membrane using the application of Fick's Law is less than the actual value of DH. In the experiments in which electrolysis is used to commence the diffusion of hydrogen the Gorsky effect contribution for diffusion seems to be less significant.

\section{References:}

Baranowski, B. and Lewis, F.A. (1989). Ber. Bunsenzes, Phys. Chem., 93: 1225.

Chen Min Li. (1978). Metal Trans. 9A: 1353-78.

Flanagan, T.B. and Lynch, J.F. (1976). J. Less Common Metals, 49: 25-29.

Kajenthini, A. (2012). Project Report submitted in partial fulfillment for the special Degree of Bachelor of Science (Physics) Department of Physics, University of Jaffna, Sri Lanka.

Kandasamy, K. (1988). Scripta Metall, 22: 479481.

\section{6}


Kandasamy, K. (1995). Int. J. Hydrogen Energy, Lewis, F.A., Kandasamy, K. and Baranowski, 20(6): 455-63.

B. (1988). Int. J. Hydrogen Energy, 13(7): 439-442.

Kandasamy, K., Lewis, F.A., Magennis, J.P., Mckee S.G. and Tong, X.Q. (1991). Z. Phys. Chem, 29: 171-213.

Lewis, F.A., Magennis, J.P., McKee, S.G. and Ssebuwufu, P.J.M. (1983). Nature, 306-673.

Kandasamy, K., Lewis, F.A. and Tong, X.Q. Sakamoto, Y., Tanaka, H., Lewis, F.A., Tong (1990). Proceedings of $4^{\text {th }}$ International Conference on Hydrogen Effects on Materials Behaviour. Ed. N. R. Moody and A. W. Thompson, Jackson Lodge WY, (Met. Soc. AIME Warrendale P.A,), 249 pp. X.Q. and Kandasamy, K. (1996). Int. J. Hydrogen Energy, 21: 1025pp.

Lewis, F.A., Baranowski, B. and Kandasamy, K. (1987). J. Less-Common Metals, 134: 27 - 